\title{
Resveratrol enhances the radiosensitivity of nasopharyngeal carcinoma cells by downregulating E2F1
}

\author{
YUHUI TAN $^{1 *}$, XIANLI WEI $^{2 *}$, WENYIN ZHANG $^{1}$, XIAOLAN WANG $^{1}$, \\ $\mathrm{KUN} \mathrm{WANG}^{3}$, BIAOYAN DU ${ }^{3}$ and JIANYONG XIAO ${ }^{1}$ \\ ${ }^{1}$ Department of Biochemistry, Guangzhou University of Chinese Medicine, Guangzhou, Guangdong 510006; \\ ${ }^{2}$ Department of Medical Instruments, Guangdong Food and Drug Vocational College, Guangzhou, Guangdong 510520; \\ ${ }^{3}$ Department of Pathology, Guangzhou University of Chinese Medicine, Guangzhou, Guangdong 510006, P.R. China
}

Received September 8, 2016; Accepted January 23, 2017

DOI: $10.3892 / o r .2017 .5413$

\begin{abstract}
Identification of safe, effective radiosensitizing agents is urgently needed to improve the outcome of radiotherapy in nasopharyngeal cancer (NPC). In this study, we assessed the ability of the polyphenol resveratrol to act as a radiosensitizer in vitro and in vivo. CNE-1 cells were treated with $50 \mu \mathrm{M}$ resveratrol for $24 \mathrm{~h}$, then irradiated. E2F transcription factor 1 (E2F1) was stably knocked down and overexpressed using lentiviruses. A xenograft model of NPC was established in nude mice using CNE-1 cells. Compared to control DMSO-treated CNE-1 cells, resveratrol inhibited colony-forming ability and induced G1 phase cell cycle arrest. Radiation survival curves confirmed resveratrol significantly sensitized CNE-1 cells, and resveratrol in combination with $2 \mathrm{~Gy}$ irradiation synergistically increased apoptosis. Immunoblotting showed resveratrol dose- and time-dependently downregulated E2F1 and phosphoAKT (p-AKT). Knockdown of E2F1 significantly increased radiosensitivity and downregulated p-AKT; overexpression of E2F1 reversed resveratrol-induced radiosensitivity and upregulated p-AKT. In vivo, $50 \mathrm{mg} / \mathrm{kg} /$ day resveratrol and 4 Gy irradiation led to significantly lower tumor volume and tumor weight compared to resveratrol or irradiation alone. Our findings show that resveratrol increases the radiosensitivity of NPC cells by downregulating E2F1 and inhibiting p-AKT, and therefore has potential as a radiosensitizer for NPC.
\end{abstract}

Correspondence to: Dr Jianyong Xiao, Department of Biochemistry, Guangzhou University of Chinese Medicine, Guangzhou, Guangdong 510006, P.R. China

E-mail: jianyongxiao@163.com

${ }^{*}$ Contributed equally

Key words: resveratrol, nasopharyngeal carcinoma, radiosensitivity, irradiation, E2F1

\section{Introduction}

Nasopharyngeal cancer (NPC) is prevalent in Southeast Asia, especially in the Cantonese region (1). Radiotherapy is the primary treatment and outcomes have improved considerably in the last decade. The 5-year overall survival rate is presently at least $85.8 \%$ for early-stage NPC $(2,3)$. However, most patients are diagnosed with advanced NPC, which has a local control rate of only 50\% and 5-year overall survival rate of $40-70 \%$ (4). Radioresistance is a key factor that contributes to local recurrence and distant metastasis in NPC. However, increasing the radiotherapy dose alone would affect anatomically-close crucial structures and reduce the quality of life (5). Moreover, present standard chemoradiation strategies have already reached the upper toxicity limits in terms of side effects and complications (6). Therefore, a novel, effective, less toxic radiosensitizer is urgently needed to improve the therapeutic outcomes of patients with NPC.

Resveratrol is a polyphenol present in a wide variety of dietary sources including grapes, peanuts and red wine, as well as plant species such as Polygonum cuspidatum and Yucca schidigera (7-9). Resveratrol exhibits many health-beneficial properties such as antioxidant and anti-inflammatory effects, and has been used as healthcare product for cardiac protection, anti-aging and lifespan extension (10-12). Apart from these traditional applications of resveratrol, studies in a wide range of cancer cell lines suggest resveratrol may also exert anticancer effects by promoting G1 or G2 phase cell cycle arrest and subsequently inducing apoptosis (13-15). A recent study showed resveratrol retarded cell cycle progression and eventually arrested NPC cells in the G0/G1 phase.

As G1 or G2 phase cells are more sensitive to irradiation than $S$ phase cells (16), we hypothesized that resveratrol may enhance radiosensitivity of NPC cells. Here, we assessed the ability of resveratrol to sensitize CNE-1 cells to irradiation both in vitro and in vivo and explored the associated molecular mechanisms.

\section{Materials and methods}

Cell culture and treatment. Human NPC CNE-1 cells were obtained from Sun Yat-Sen University Cell Collection and 
cultured in RPMI-1640 (Invitrogen Life Technologies, Carlsbad, CA, USA) supplemented with $10 \%$ fetal bovine serum (FBS; Hyclone, Logan, UT, USA) and $100 \mathrm{U} / \mathrm{ml}$ penicillin plus streptomycin (Life Technologies, Gaithersburg, $\mathrm{MD}$, USA). Cells were irradiated at $12.7 \mathrm{~Gy} / \mathrm{min}$ at room temperature using an RS 2000 X-ray Biological Irradiator (Rad Source Technologies, Inc., Suwanee, GA, USA). Cells were treated as follows: control [dimethyl sulfoxide (DMSO)]; resveratrol $(0-150 \mu \mathrm{M}$ resveratrol dissolved in DMSO, no. R5010; Sigma-Aldrich, St. Louis, MO, USA); irradiation (0-8 Gy irradiation); and resveratrol + irradiation (pretreatment with resveratrol for $24 \mathrm{~h}$ followed by irradiation).

Cell viability assay. CNE-1 cells were seeded into 96-well plates in triplicate $(3,000$ cells/well), cultured for $24 \mathrm{~h}$, then treated with resveratrol for 24,48 or $72 \mathrm{~h}$. Cell viability was measured using the 3-(4,5-dimethylthiazol-2-yl)-2,5-diphenyltetrazolium bromide (MTT; Sigma-Aldrich) assay. Absorbance values were measured at $490 \mathrm{~nm}$ using a microplate reader (Bio-Tek ELx800; Bio-Tek Instruments, Inc., Winooski, VT, USA).

Radiation cell survival assay. CNE-1 cells were seeded into 6 -well plates $\left(5 \times 10^{2}\right.$ cells/well), incubated overnight, treated with resveratrol and/or irradiation as described above, then the media was removed and replaced with fresh resveratrol-free culture medium. After 15 days, the cells were fixed in ice-cold methanol, stained with Giemsa solution, photographed and the numbers of colonies containing at least 50 cells were scored. Cell survival curves were fitted using the linear-quadratic formula: surviving fraction $=\mathrm{e}^{\left(-\alpha \mathrm{D}-\beta \mathrm{D}^{2}\right)}(17)$; $\mathrm{D}$ represents the dose of irradiation; $\alpha$ and $\beta$ are radibiological cell survival parameters.

Cell cycle and apoptosis analysis. CNE-1 cells $\left(5 \times 10^{4}\right.$ cells/well) in 6-well plates were treated as described above, trypsinized, fixed and stained according to the instructions of the Cell Cycle and Apoptosis kit (no. 559763; BD Biosciences, Franklin Lakes, NJ, USA). Cell cycle distribution and apoptosis were analyzed using a Cytomics $^{\mathrm{TM}}$ FC500 flow cytometer and CXP analysis software (both from Beckman-Coulter, Anacortes, WA, USA).

Immunoblotting analysis. CNE-1 cells were treated in 6-well plates, lysed in RIPA buffer ( $0.25 \mathrm{M}$ Tris-HCl, $\mathrm{pH} 6.8,8 \%$ SDS, $1 \mathrm{mM}$ phenylmethylsulfonyl fluoride, $10 \mathrm{mg} / \mathrm{ml}$ aprotinin and $1.0 \mathrm{mg} / \mathrm{ml}$ leupeptin). The cell extracts were separated by $10 \%$ SDS-PAGE, transferred onto PVDF membranes (Millipore, Bedford, MA, USA) and immunoblotted using anti-E2F transcription factor 1 (anti-E2F1) (no. ab179445), anti-pan-AKT (phospho T308, no. ab38449), anti-pan-AKT (no. ab64148), anti-ERK1 (pT202/pY204) + ERK2 (pT185/pY187, no. ab50011), anti-ERK1 + ERK2 (no. ab17942) and anti-GAPDH (no. ab181602) (all from Abcam, Cambridge, $\mathrm{UK})$.

Recombinant lentivirus construction and transduction. We inserted the sequence: 5'-GTCACGCTATGAGACCTCA-3' into the LV008 lentiviral silencing vector (Forevergen, Guangzhou, China) to express a short hairpin RNA (shRNA) in order to effectively knock down human E2F1. To overexpress E2F1, we subcloned the E2F1 gene (GenBank accession no. GI: 168480109) into the LV003 lentiviral expression vector (Forevergen). The recombinant lentiviruses (and corresponding negative controls) were packaged in 293T cells and used to create stable CNE-1 cells. The expression of E2F1 in the stable CNE-1 cell lines was validated by immunoblotting.

Animals. Forty specific pathogen-free BALB/c nude mice (male, 6-weeks-old) were purchased from Vital River Laboratory Animal Technology Co., Ltd. (Peking, China). All protocols were approved by the Animal Experimentation Ethics Committee of Guangzhou University of Chinese Medicine and in compliance with recommended NIH guidelines for Care and Use of Animals for scientific purposes.

In vivo experiments. $\mathrm{CNE}-1$ cells $\left(5 \times 10^{6}\right)$ in the exponential phase of growth were subcutaneously injected into the right flank of BALB/c mice (day 0). The volumes of the xenograft tumors were measured every 4 days. When the tumors reached $100 \mathrm{~mm}^{3}$ (day 8), the mice were randomly assigned to four groups ( $\mathrm{n}=10$ mice each) and treated as follows: vehicle (5\% ethanol and 25\% polyethylene glycol 400 in distilled water); vehicle and $4 \mathrm{~Gy}$ irradiation; resveratrol $(50 \mathrm{mg} / \mathrm{kg} / \mathrm{day})$; and resveratrol $(50 \mathrm{mg} / \mathrm{kg} /$ day) plus 4 Gy irradiation. On day 8 , mice were treated intraperitoneally with resveratrol or vehicle until the completion of the experiment. On day 12 , mice were irradiated once a day for consecutive 3 days. Mice were sacrificed on day 28 to measure tumor volume and tumor weight.

Statistical analysis. All data are mean \pm standard deviation. SPSS 13.0 software was used for statistical analyses (SPSS, Inc., Chicago, IL, USA). Each in vitro experiment was repeated independently three times. Differences between groups were compared using the Student's t-test; one-way analysis of variance (ANOVA) was used for multiple comparisons. Two-tailed $\mathrm{P}$-values $<0.05$ were considered statistically significant.

\section{Results}

Resveratrol reduces the viability of CNE-1 NPC cells. To investigate the effect of resveratrol on the radiosensitivity of NPC cells, we first optimized the resveratrol dose and treatment time. The MTT assay showed exposure to $<100 \mu \mathrm{M}$ resveratrol for $24 \mathrm{~h}$ had no significant effect on CNE-1 cell viability (Fig. 1A). Next, we treated CNE-1 cells with 25, 50 or $100 \mu \mathrm{M}$ resveratrol for $24 \mathrm{~h}$ and assessed cell proliferation using the colony formation assay and cell cycle analysis. Resveratrol dose-dependently decreased colony formation and induced cell cycle G1 arrest (Fig. 1B and C). Notably, $50 \mu \mathrm{M}$ resveratrol significantly inhibited the proliferation of CNE-1 cells. Therefore, we pretreated CNE-1 cells with $50 \mu \mathrm{M}$ resveratrol for $24 \mathrm{~h}$ in all subsequent experiments.

Resveratrol enhances the radiosensitivity of CNE-1 NPC cells. CNE-1 cells seeded in 6-well plates were treated with resveratrol for $24 \mathrm{~h}$, then switched to media without resveratrol, irradiated at 2, 4 or $6 \mathrm{~Gy}$ and cultured for 15 days. Colony formation assays showed the survival rates of the cells treated with resveratrol and irradiation decreased significantly as the dose of 
A

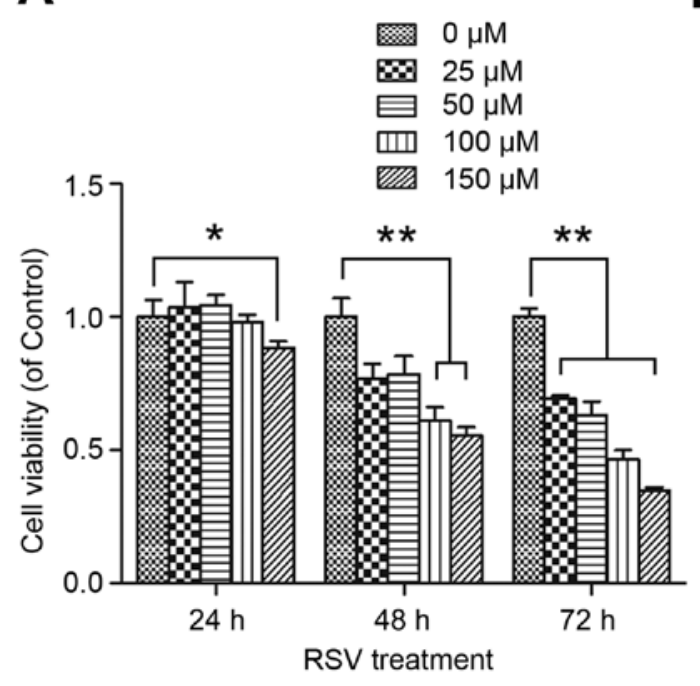

B
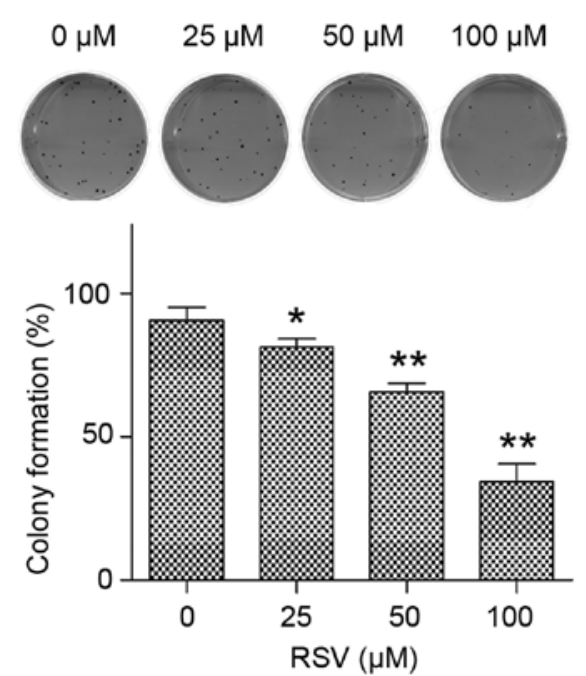

$\operatorname{RSV}(25 \mu \mathrm{M})$
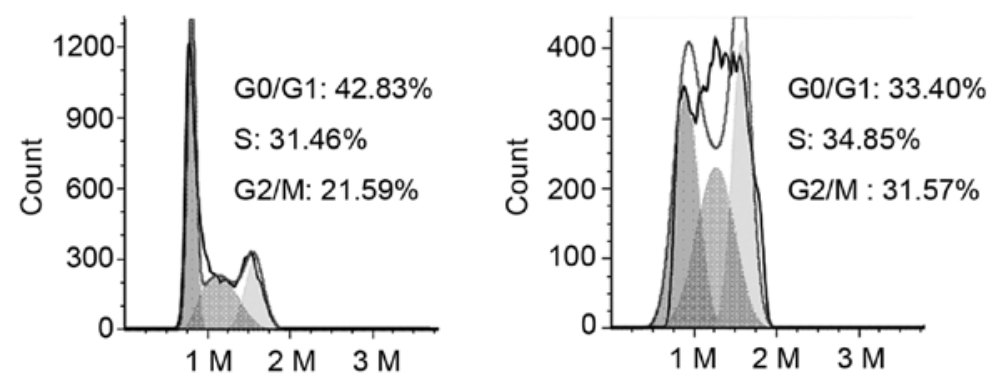

$\operatorname{RSV}(50 \mu \mathrm{M})$
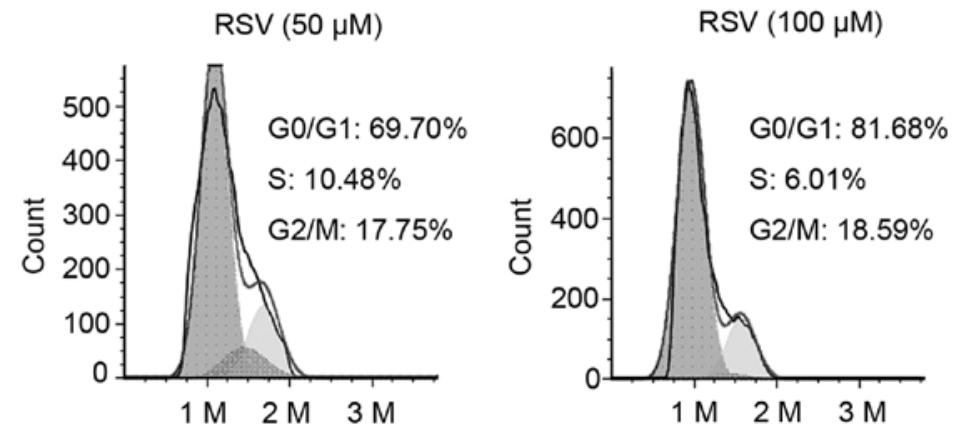

Figure 1. RSV reduces the viability and proliferation of NPC cells. (A) Effect of RSV on the viability of CNE-1 cells. CNE-1 cells were exposed to $0,25,50$, 100 or $150 \mu \mathrm{M}$ RSV for 24,48 or $72 \mathrm{~h}$, then cell viability was assessed using the MTT assay. (B and C) Effect of RSV on the proliferation of CNE-1 cells. CNE-1 cells were treated with $0,25,50$ or $100 \mu \mathrm{M}$ RSV for $24 \mathrm{~h}$, then analyzed using (B) the colony formation assay and (C) flow cytometric cell cycle analysis. ${ }^{*} \mathrm{P}<0.05 ;{ }^{* *} \mathrm{P}<0.01$. RSV, resveratrol; NPC, nasopharyngeal cancer.

radiation increased, compared to cells treated with irradiation alone ( $\mathrm{P}<0.05$; Fig. 2A). We applied the linear-quadratic model to fit radiosensitivity curves for each group; resveratrol-treated cells were more sensitive to irradiation than untreated cells [survival rate after irradiation with 2 Gy (SF2): $65.01 \pm 6.78 \%$ vs. $78.01 \pm 4.89 \%, \mathrm{P}=0.03$; Fig. $2 \mathrm{~B}$ ]. Radiosensitivity can also be evaluated using the radiobiological parameters $\alpha$ derived from the linear-quadratic formula. The $\alpha$-value indicates radiosensitivity. When resveratrol-treated and untreated cells were compared, the $\alpha$-value increased $(0.09 \pm 0.01$ vs. $0.18 \pm 0.02, \mathrm{P}=0.004)$, demonstrating pretreatment with resveratrol enhanced the radiosensitivity of NPC cells.
To further assess the effects of resveratrol and/or irradiation on NPC cells, CNE-1 cells treated as described above were stained using Annexin V/PI. Flow cytometric analysis showed (Fig. 2C) resveratrol did not significantly increase the apoptotic rate compared to control cells. Although irradiation alone induced an apoptotic rate of $15 \%$, the apoptotic rate of CNE-1 irradiated cells pretreated with resveratrol was $\sim 30 \%$ ( $\mathrm{P}<0.01)$, indicating that pretreatment with resveratrol enhances the cell-killing effect of irradiation in NPC cells.

Resveratrol downregulates E2F1 in CNE-1 NPC cells. As resveratrol dose-dependently induced $\mathrm{G} 0 / \mathrm{G} 1$ cell cycle 

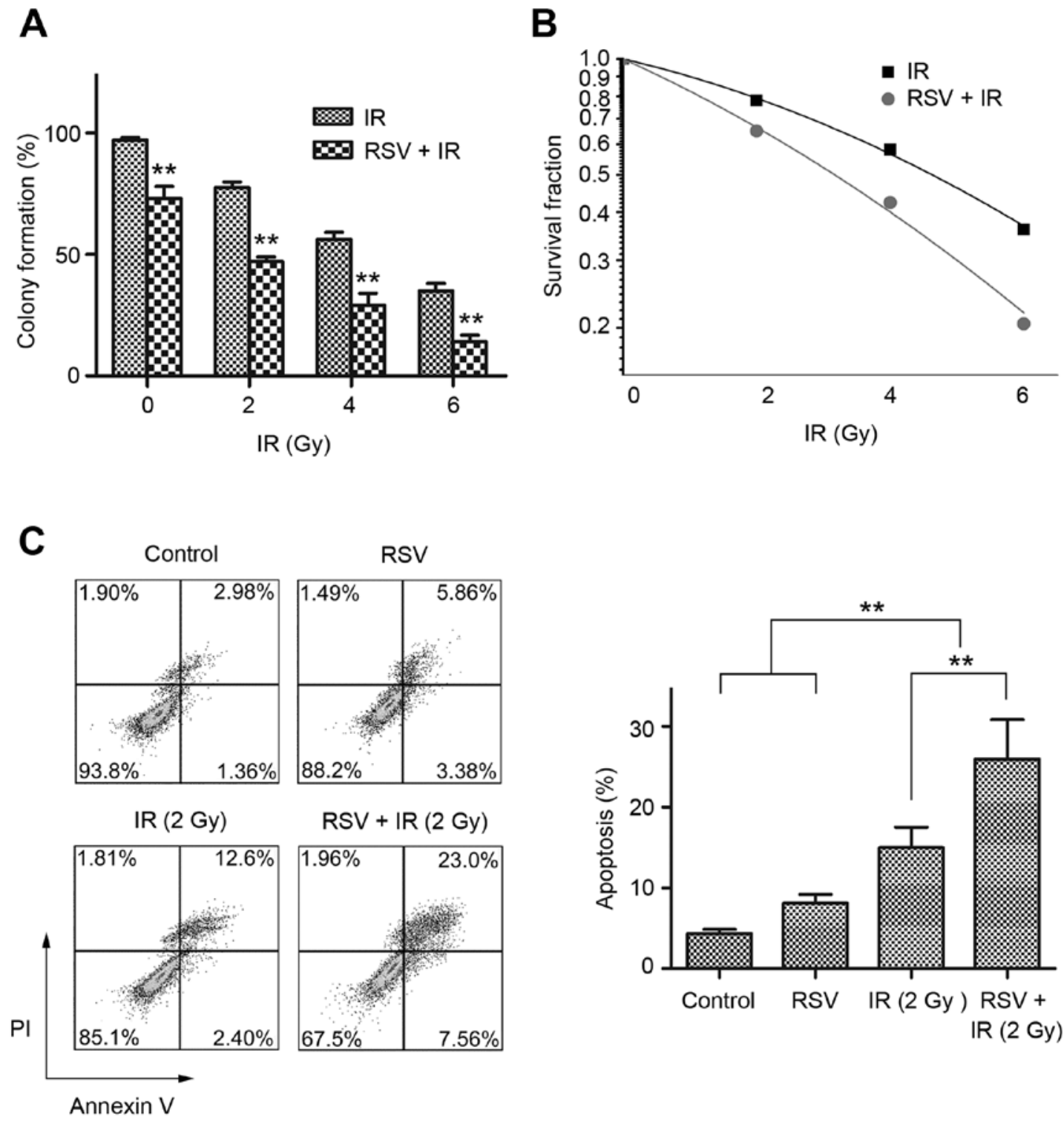

Figure 2. RSV enhances the radiosensitivity of NPC cells in vitro. CNE-1 cells were treated with vehicle (DMSO) or $50 \mu \mathrm{M}$ RSV for $24 \mathrm{~h}$ and then irradiated (IR) at 0, 2, 4 or $6 \mathrm{~Gy}$. (A) Effect of RSV and/or irradiation on colony-forming ability. (B) Radiation survival curves for CNE-1 cells fitted using the linear-quadratic model. (C) The cell-killing effect of RSV and/or 2 Gy irradiation, as analyzed by Annexin V/PI double staining and flow cytometry. ${ }^{* *} \mathrm{P}<0.01$. RSV, resveratrol; NPC, nasopharyngeal cancer.

arrest, we next examined the expression of E2F1, a major protein that regulates G0/G1 phase (18). CNE-1 cells were pretreated with resveratrol for $24 \mathrm{~h}$ then irradiated at 0,2 or 4 Gy. Immunoblotting revealed resveratrol downregulated E2F1 in a dose- and time-dependent manner (Fig. 3A) in both non-irradiated and irradiated cells (Fig. 3B). Notably, p-AKT was simultaneously downregulated by resveratrol, though the expression of p-ERK did not change. These results suggest resveratrol may inhibit p-AKT by downregulating E2F1.

Knockdown of E2F1 enhances the radiosensitivity of CNE-1 NPC cells. To elucidate whether E2F1 regulates the radiosensitivity of NPC cells, we knocked down E2F1 using a lentiviral system; immunoblotting confirmed E2F1 was downregulated and furthermore, $\mathrm{p}$-AKT was concomitantly reduced (Fig. 4A). As E2F1 regulates the G0/G1 progression of the cell cycle (18), we performed cell cycle analysis by PI staining and flow cytometry. Stable knockdown of E2F1 (CNE-1-shE2F1) increased the percentage of cells in the G0/G1 phase compared to control CNE-1 cells (CNE-1-NC). Furthermore, the colony formation assay indicated that the depletion of E2F1 reduced the colony formation rate (data not shown), indicating E2F1 plays an important role in the proliferation of NPC cells (Fig. 4B).

Next, CNE-1-NC and CNE-1-shE2F1 cells were seeded in 6 -well plates, treated with or without resveratrol, irradiated at $0,2,4$ or $6 \mathrm{~Gy}$, cultured for 15 days, then subjected to the colony formation assay (Fig. 4A). Based on the clone formation rate, we applied the linear-quadratic model to fit radiosensitivity curves for each group (Fig. 4B). CNE-1-shE2F1 cells had a higher $\alpha$-value $(0.22 \pm 0.03$ vs. $0.10 \pm 0.01, \mathrm{P}=0.002)$ and lower SF2 $(60.45 \pm 3.24 \%$ vs. $79.51 \pm 5.42 \%, \mathrm{P}=0.03)$ than control cells, demonstrating knockdown of E2F1 enhanced the radiosensitivity of CNE-1 cells. 

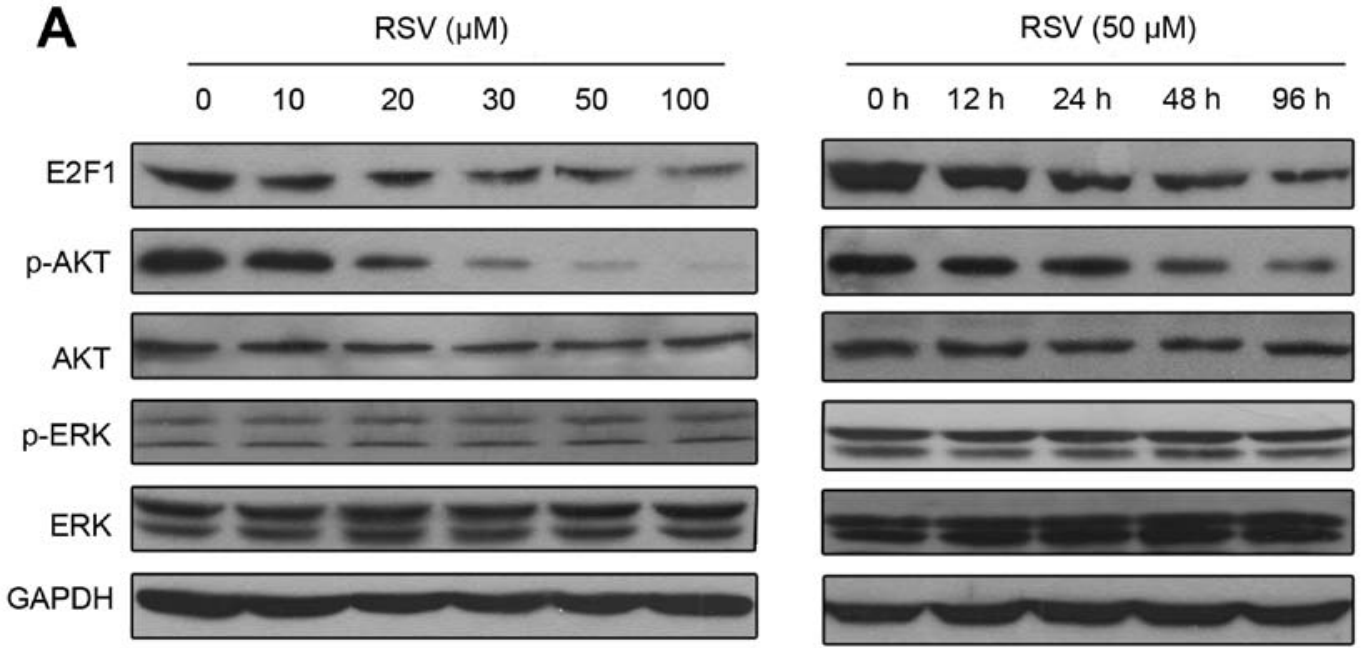

B

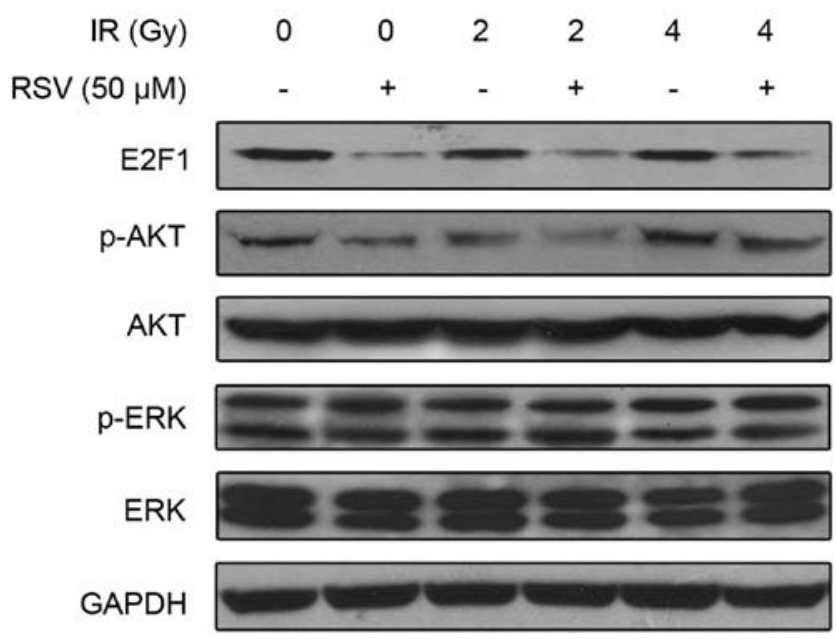

Figure 3. RSV downregulates E2F1 in NPC cells. (A) Immunoblotting analysis of E2F1, AKT and ERK expression in CNE-1 cells after RSV treatment and/or IR. (B) Immunoblotting showed resveratrol dose- and time-dependently downregulated E2F1 and p-AKT. RSV, resveratrol; E2F1, E2F transcription factor 1; NPC, nasopharyngeal cancer; IR, irradiation.

CNE-1-NC and CNE-1-shE2F1 were seeded in 6-well plates, irradiated at $2 \mathrm{~Gy}$ and cultured for $72 \mathrm{~h}$. Annexin V/PI double staining and flow cytometric analysis (Fig. 4C) revealed CNE-1-shE2F1 cells had a significantly higher apoptotic rate than CNE-1-NC cells. Collectively, the data indicate that knockdown of E2F1 enhanced the cell-killing effect of irradiation in NPC cells.

Overexpression of E2F1 reverses resveratrol-induced radiosensitivity in CNE-1 NPC cells. To confirm if E2F1 is involved in the resveratrol-induced radiosensitivity of CNE-1 cells, we established an E2F1-overexpressing stable CNE-1 cell line (CNE-1-E2F1) and control cells (CNE-1-NC) using a lentiviral system. Immunoblotting revealed E2F1 was overexpressed in CNE-1-E2F1 cells cultured in resveratrol-free media and resveratrol for $24 \mathrm{~h}$ compared to the respective CNE-1-NC cells (Fig. 5A). The colony formation assay and cell cycle analysis demonstrated resveratrol decreased the colony formation rate and induced G0/G1 cell cycle arrest in CNE-1-NC cells, whereas overexpression of E2F1 reversed the ability of resveratrol to inhibit colony formation and induce cell cycle arrest in CNE-1-E2F1 cells (data not shown).

Next, we pretreated CNE-1-NC and CNE-1-E2F1 cells seeded in 6-well plates with resveratrol for $24 \mathrm{~h}$, irradiated the cells at $0,2,4$ and $6 \mathrm{~Gy}$, and then returned to the incubator for 15 days in resveratrol-free culture media. Based on the colony formation rate, we applied the linear-quadratic model to plot radiosensitivity curves (Fig. 5B). Compared to CNE-1-NC cells, CNE-1-E2F1 cells had a lower $\alpha$-value $(0.13 \pm 0.02$ vs. $0.23 \pm 0.01, \mathrm{P}=0.0006)$ and higher $\mathrm{SF} 2$ value $(73.01 \pm 3.48 \%$ vs. $59.94 \pm 6.05 \%, \mathrm{P}=0.01)$, demonstrating overexpression of $\mathrm{E} 2 \mathrm{~F} 1$ reversed resveratrol-induced radiosensitivity.

Finally, CNE-1-NC and CNE-1-E2F1 were seeded into 6-well plates, pretreated with resveratrol for $24 \mathrm{~h}$, irradiated at $2 \mathrm{~Gy}$, and then cultured for $72 \mathrm{~h}$. Annexin V/PI double staining and flow cytometry showed CNE-1-E2F1 cells had a significantly higher rate of apoptosis than CNE-1-NC cells (Fig. 5C), indicating that overexpression of E2F1 impaired the cell-killing effect of resveratrol plus irradiation in CNE-1 cells. 


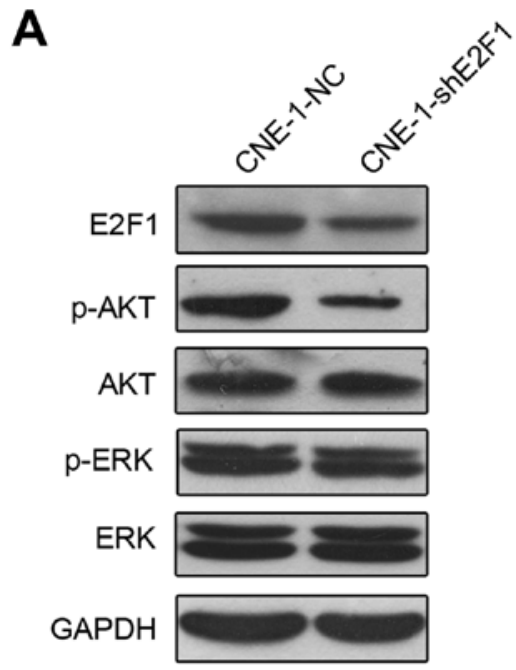

B

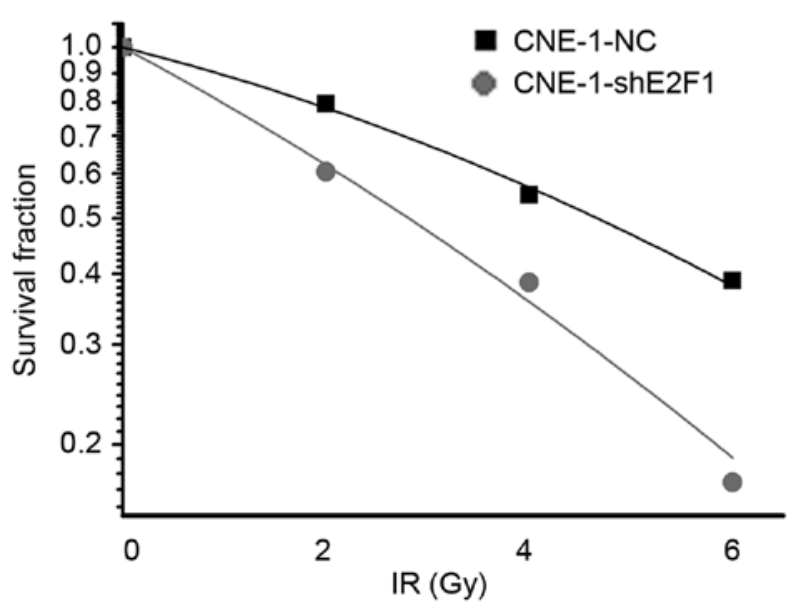

C

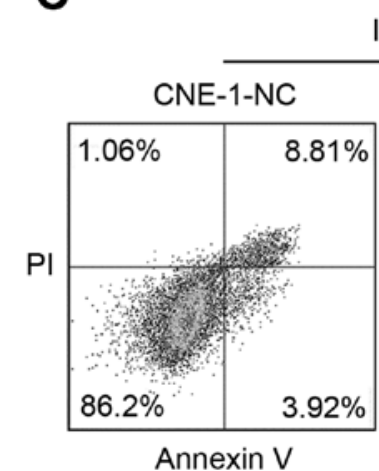

IR (2Gy)

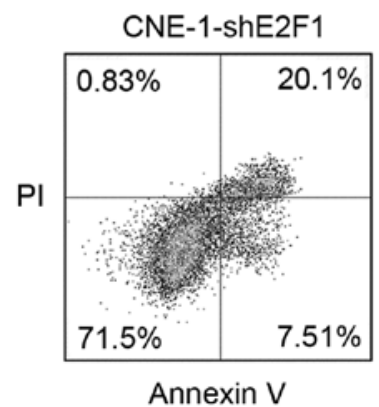

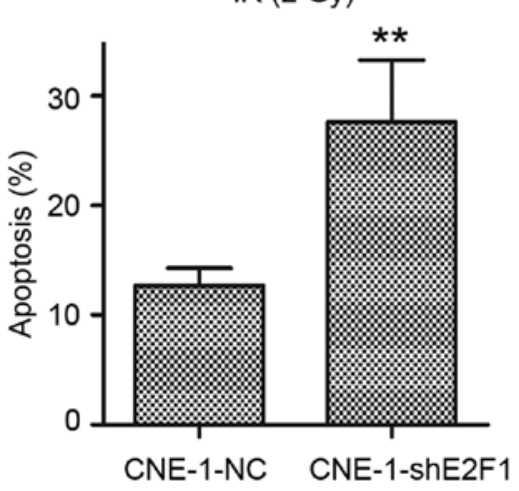

Figure 4. Stable knockdown of E2F1 enhances the radiosensitivity of NPC cells. (A) Compared to negative control CNE-1 cells stably expressing empty lentivirus (CNE-1-NC), immunoblotting indicated E2F1 was stably knocked down in CNE-1-shE2F1 cells and p-AKT was concomitantly downregulated. (B and C) CNE-1-NC and CNE-1-shE2F1 were exposed to 0,2,4 or 6 Gy IR. (B) Radiation survival curves based on the colony formation assay. (C) Annexin V/PI double staining and flow cytometric analysis of the rate of apoptosis. ${ }^{* *} \mathrm{P}<0.01$. E2F1, E2F transcription factor 1; NPC, nasopharyngeal cancer; IR, irradiation.

Resveratrol and irradiation synergistically inhibit NPC xenograft tumor growth in vivo. To assess resveratrol-induced radiosensitivity in vivo, we established a xenograft model of NPC in BALB/c nude mice. When the volume of tumors reached $\sim 0.1 \mathrm{~cm}^{3}$ (day 8), the mice were intraperitoneally injected with resveratrol $(50 \mathrm{mg} / \mathrm{kg} /$ day $)$ or vehicle until the completion of the experiment. On day 12, tumor-bearing mice were irradiated ( $4 \mathrm{~Gy} /$ day) for consecutive 3 days. On sacrifice (day 28), the weight of the mice was not significantly different between groups (data not shown).

Compared to the control group, tumor-bearing nude mice exposed to irradiation alone exhibited a $33 \%$ reduction in tumor volume $\left(1360.69 \pm 188.45\right.$ vs. $\left.2034.86 \pm 112.96 \mathrm{~mm}^{3}, \mathrm{P}<0.01\right)$ and $32 \%$ decrease in tumor weight $(1213.75 \pm 163.87$ vs. $1789.44 \pm 101.85 \mathrm{mg}, \mathrm{P}<0.01$; Fig. 6). Although resveratrol alone had a certain antitumor effect, the combinatorial therapy of resveratrol and irradiation resulted in a $74 \%$ reduction in tumor volume $\left(520.13 \pm 68.51\right.$ vs. $2034.86 \pm 112.96 \mathrm{~mm}^{3}$, $\mathrm{P}<0.01)$ and $73 \%$ reduction in tumor weight $(479.22 \pm 62.84$ vs. $1789.44 \pm 101.85 \mathrm{mg}, \mathrm{P}<0.01$; Fig. 6 ) compared to the control group. These results indicate that administration of resveratrol enhanced the radiosensitivity of the NPC xenograft tumors in vivo.

\section{Discussion}

Radioresistance can lead to local recurrence and distant metastasis and is associated with a poor prognosis, especially in advanced stage NPC (19). Due to its antioxidant and anti-aging properties, resveratrol has become a popular healthcare product for cardiovascular protection $(20,21)$. This study demonstrated that resveratrol enhances the radiosensitivity of NPC cells in vitro and in vivo via a mechanism involving E2F1 and its downstream target p-AKT. Therefore, resveratrol combined with irradiation may represent a promising strategy to further improve the outcomes of patients with NPC.

Resveratrol is reported to exert a diverse range of effects, low concentrations $(0.1-2.5 \mu \mathrm{M})$ promoted the viability and proliferation of human umbilical cord mesenchymal stem cells while higher concentrations had the opposite effects (22). High-dose resveratrol also exerts cytotoxic effects in a variety 
A

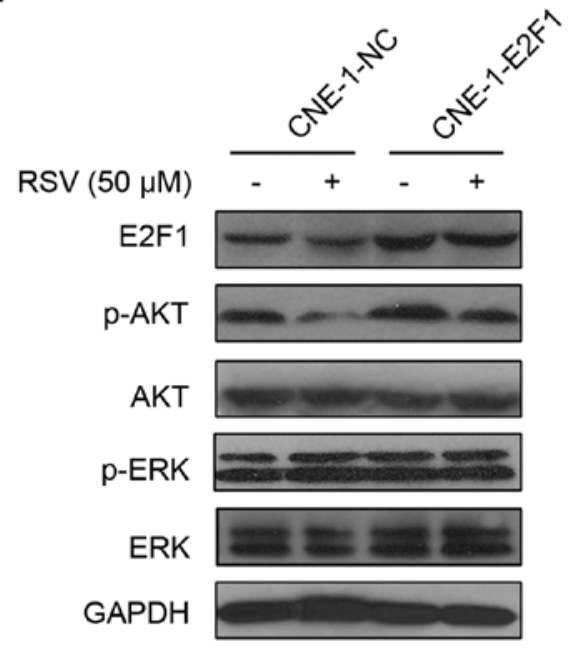

B

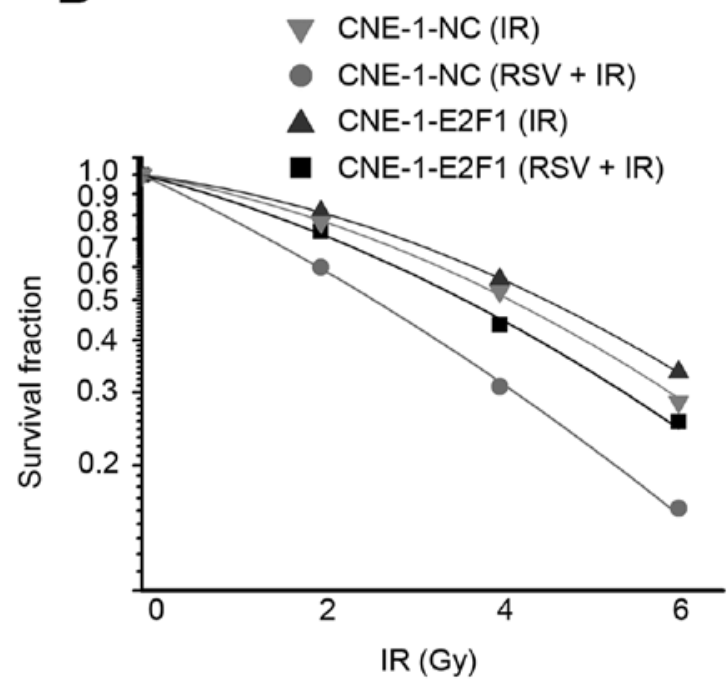

C
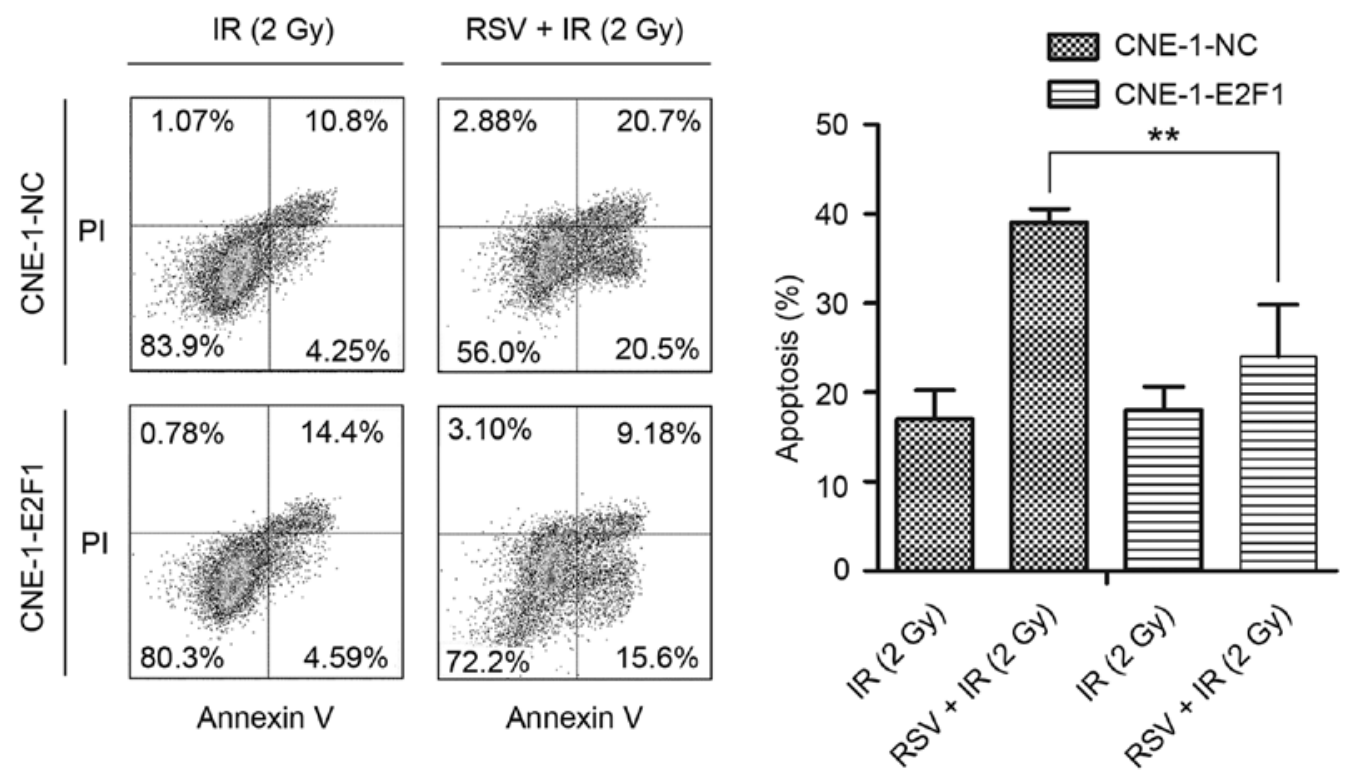

Figure 5. Overexpression of E2F1 reverses resveratrol-induced radiosensitivity in NPC cells. (A) CNE-1 cells stably overexpressing E2F1 (CNE-1-E2F1) and negative control cells (CNE-1-NC) were treated with or without RSV for $24 \mathrm{~h}$. Immunoblotting confirmed the overexpression of E2F1 in CNE-1-E2F1 cells. (B and C) CNE-1-NC and CNE-1-E2F1 were pretreated with RSV for $24 \mathrm{~h}$ then exposed to $0,2,4$ or 6 Gy IR. (B) Radiation survival curves for CNE-1 cells based on the colony formation assay. (C) Annexin V/PI double staining and flow cytometric analysis of the rate of apoptosis. "* P<0.01. E2F1, E2F transcription factor 1; NPC, nasopharyngeal cancer; RSV, resveratrol; IR, irradiation.

of cancer cell lines $(23,24)$. However, to achieve effective and desirable outcomes with minimum toxicity to normal cells, a clinical resveratrol and irradiation regime would need to be designed very carefully. Although different types of cancer cells respond to resveratrol and irradiation in a varied manner, the existing studies all suggested that the highest concentration of $100 \mathrm{mM}$ resveratrol and highest dose of 5 Gy irradiation are sufficient to kill cells by induction of apoptosis (25). In this study, we pretreated CNE-1 NPC cells with $50 \mu \mathrm{M}$ resveratrol for $24 \mathrm{~h}$ before irradiation. This moderate dose of resveratrol did not affect the cell viability of CNE-1 cells, but did significantly reduce the proportion of $\mathrm{S}$ phase cells. Therefore, this non-toxic dose of resveratrol may have the potential to increase the radiosensitivity of NPC cells, as S phase cells normally exhibit resistance to irradiation therapy (16).

Resveratrol and irradiation have been reported to modulate target molecules that inhibit proliferation and induce apoptosis. A number of proliferation or apoptosis-related molecules such as cyclins, p53, p21, NF- $\kappa \mathrm{B}$ and Bcl-2 have been reported to be involved in the resveratrol-mediated radiosensitivity of cancer cells (26-28).

This study showed resveratrol could also arrest NPC cells in the G0/G1 phase. Therefore, we examined the expression of E2F1, a key molecule that regulates the G0/G1 progression (18). Resveratrol downregulated E2F1 in CNE-1 cells in a dose- and 
A

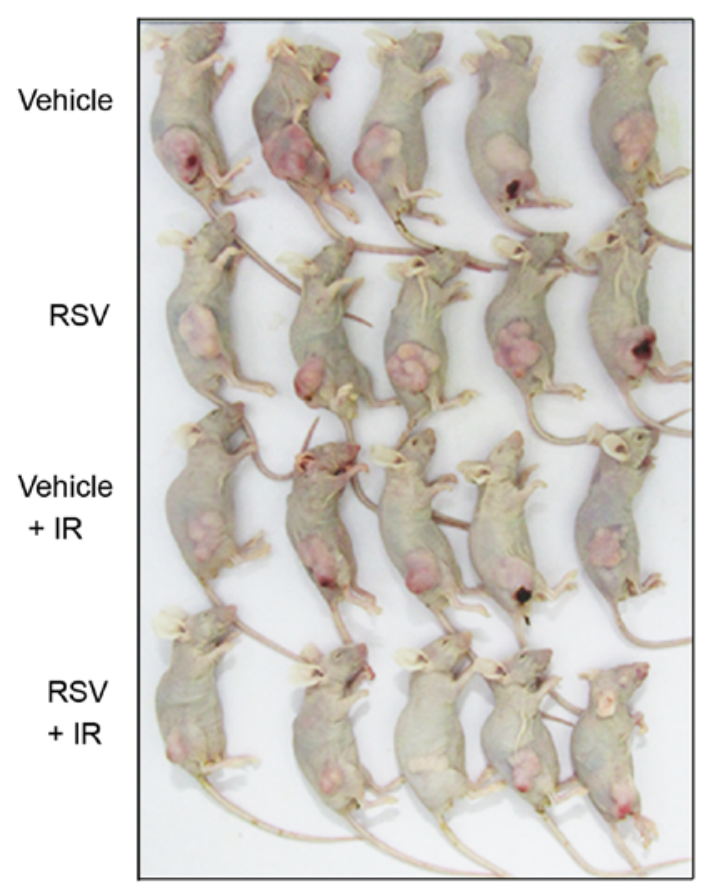

C
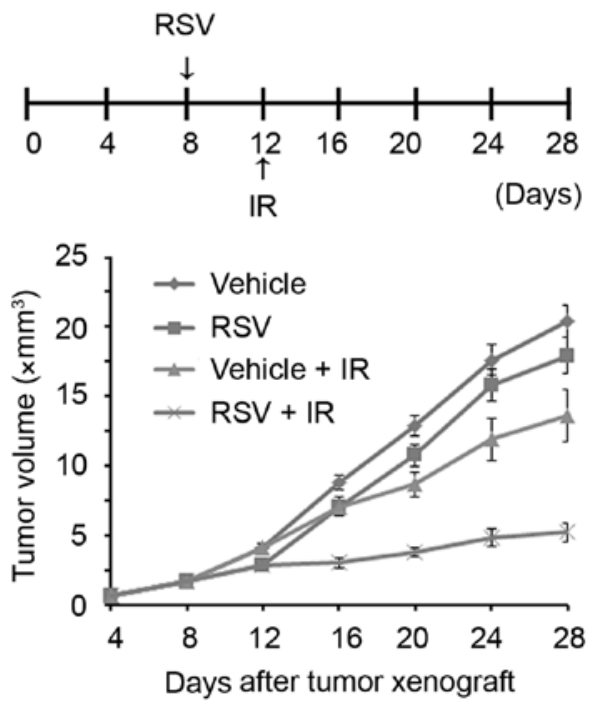

D

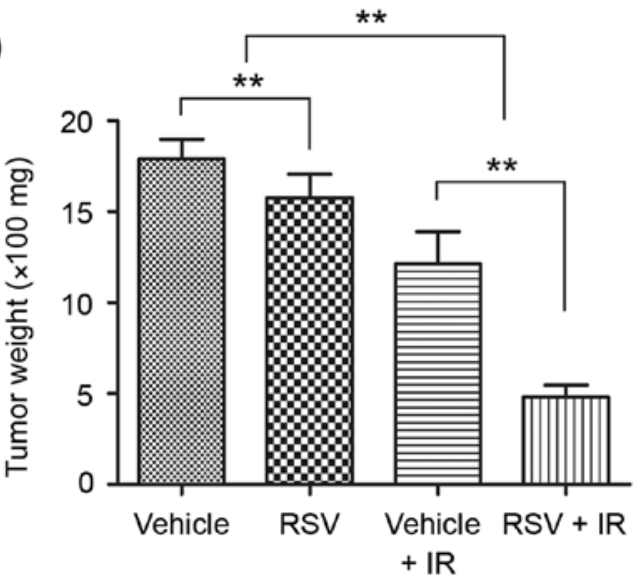

Figure 6. RSV and irradiation synergistically inhibit NPC tumor xenograft growth in vivo. Xenograft tumors were established by subcutaneous injection of CNE-1 cells into the right flank of BALB/c mice. Mice were randomly assigned (n=10/group) to the vehicle group, vehicle and IR (4 Gy) group, RSV ( $50 \mathrm{mg} / \mathrm{kg} /$ day) group and RSV (50 mg/kg/day) plus 4 Gy IR group. (A and B) Images of the (A) tumor-bearing mice and (B) excised tumors. (C, upper part) The animals were treated and euthanized on day 28 of treatment. $\left(\mathrm{C}\right.$, lower part) Average tumor volume $\left(\mathrm{mm}^{3}\right)$ measured every 4 days for each group is shown. (D) Average tumor weight of the excised tumors (mg) for each group on day 28. Data are mean \pm standard deviation. ${ }^{* *} \mathrm{P}<0.01$. RSV, resveratrol; NPC, nasopharyngeal cancer; IR, irradiation 1.

time-dependent manner. Furthermore, knocking down E2F1 using RNA interference inhibited proliferation and increased the sensitivity of CNE-1 cells to irradiation (Fig. 4). Conversely, overexpression of E2F1 reversed resveratrol-induced radiosensitivity (Fig. 5), demonstrating E2F1 is required to mediate resveratrol-induced radiosensitivity in CNE-1 cells. E2F1 regulates AKT and ERK (29,30). Immunoblotting revealed resveratrol upregulated $\mathrm{p}-\mathrm{AKT}$, but not $\mathrm{p}$-ERK, indicating E2F1 modulates resveratrol-induced radiosensitivity in NPC cells by activating the AKT pathway. Further investigation is required to confirm this observation.

In conclusion, we demonstrated that a moderate dose of resveratrol sensitizes NPC cells to irradiation both in vitro and in vivo. Furthermore, E2F1 and its downstream target p-AKT are major regulators of resveratrol-induced radiosensitivity. This fundamental research provides a starting point for further studies to assess the clinical application of resveratrol as a radiosensitizer in order to improve the treatment outcomes of radiotherapy in patients with NPC.

\section{Acknowledgements}

This study was supported by grants from the National Natural Science Foundation of China (no. 81274145) and the Ministry of Education, Science Technology Development Center (no. 20124425120012). 


\section{References}

1. Jia WH, Huang QH, Liao J, Ye W, Shugart YY, Liu Q, Chen LZ, Li YH, Lin X, Wen FL, et al: Trends in incidence and mortality of nasopharyngeal carcinoma over a 20-25 year period (1978/1983-2002) in Sihui and Cangwu counties in southern China. BMC Cancer 6: 178, 2006.

2. Su SF, Han F, Zhao C, Chen CY, Xiao WW, Li JX and Lu TX: Long-term outcomes of early-stage nasopharyngeal carcinoma patients treated with intensity-modulated radiotherapy alone. Int J Radiat Oncol Biol Phys 82: 327-333, 2012.

3. Xiao WW, Han F, Lu TX, Chen CY, Huang Y and Zhao C: Treatment outcomes after radiotherapy alone for patients with early-stage nasopharyngeal carcinoma. Int J Radiat Oncol Biol Phys 74: 1070-1076, 2009.

4. Zhou J, Wang L, Xu X, Tu Y, Qin S and Yin Y: Antitumor activity of Endostar combined with radiation against human nasopharyngeal carcinoma in mouse xenograft models. Oncol Lett 4: 976-980, 2012.

5. Lee AW, Ng WT, Hung WM, Choi CW, Tung R, Ling YH, Cheng PT, Yau TK, Chang AT, Leung SK, et al: Major late toxicities after conformal radiotherapy for nasopharyngeal carcinoma-patient- and treatment-related risk factors. Int J Radiat Oncol Biol Phys 73: 1121-1128, 2009.

6. Akervall J, Nandalur S, Zhang J, Qian CN, Goldstein N, Gyllerup P, Gardinger Y, Alm J, Lorenc K, Nilsson K, et al: A novel panel of biomarkers predicts radioresistance in patients with squamous cell carcinoma of the head and neck. Eur J Cancer 50: 570-581, 2014

7. Arichi H, Kimura Y, Okuda H, Baba K, Kozawa M and Arichi S: Effects of stilbene components of the roots of Polygonum cuspidatum Sieb. et Zucc. on lipid metabolism. Chem Pharm Bull (Tokyo) 30: 1766-1770, 1982.

8. Jang M, Cai L, Udeani GO, Slowing KV, Thomas CF, Beecher CW, Fong HH, Farnsworth NR, Kinghorn AD, Mehta RG, et al: Cancer chemopreventive activity of resveratrol, a natural product derived from grapes. Science 275: 218-220, 1997

9. Soleas GJ, Diamandis EP and Goldberg DM: Resveratrol: a molecule whose time has come? and gone? Clin Biochem 30: 91-113, 1997.

10. Bradamante S, Barenghi L and Villa A: Cardiovascular protective effects of resveratrol. Cardiovasc Drug Rev 22 $169-188,2004$

11. Das S and Das DK: Anti-inflammatory responses of resveratrol. Inflamm Allergy Drug Targets 6: 168-173, 2007.

12. de la Lastra CA and Villegas I: Resveratrol as an anti-inflammatory and anti-aging agent: mechanisms and clinical implications. Mol Nutr Food Res 49: 405-430, 2005.

13. Wu Z, Liu B, E C, Liu J, Zhang Q, Liu J, Chen N, Chen R and Zhu R: Resveratrol inhibits the proliferation of human melanoma cells by inducing G1/S cell cycle arrest and apoptosis. Mol Med Rep 11: 400-404, 2015.

14. Yu XD, Yang JL, Zhang WL and Liu DX: Resveratrol inhibits oral squamous cell carcinoma through induction of apoptosis and G2/M phase cell cycle arrest. Tumour Biol 37: 2871-2877, 2016.
15. Quoc Trung L, Espinoza JL, Takami A and Nakao S: Resveratrol induces cell cycle arrest and apoptosis in malignant NK cells via JAK2/STAT3 pathway inhibition. PLoS One 8: e55183, 2013.

16. Pawlik TM and Keyomarsi K: Role of cell cycle in mediating sensitivity to radiotherapy. Int J Radiat Oncol Biol Phys 59: 928-942, 2004.

17. Fowler JF: The linear-quadratic formula and progress in fractionated radiotherapy. Br J Radiol 62: 679-694, 1989.

18. Wong JV, Dong P, Nevins JR, Mathey-Prevot B and You L: Network calisthenics: control of E2F dynamics in cell cycle entry. Cell Cycle 10: 3086-3094, 2011.

19. Lee AW, Sze WM, Au JS, Leung SF, Leung TW, Chua DT, Zee BC, Law SC, Teo PM, Tung SY, et al: Treatment results for nasopharyngeal carcinoma in the modern era: the Hong Kong experience. Int J Radiat Oncol Biol Phys 61: 1107-1116, 2005.

20. Das DK and Maulik N: Resveratrol in cardioprotection: a therapeutic promise of alternative medicine. Mol Interv 6: 36-47, 2006.

21. Pervaiz S and Holme AL: Resveratrol: its biologic targets and functional activity. Antioxid Redox Signal 11: 2851-2897, 2009.

22. Wang X, Ma S, Meng N, Yao N, Zhang K, Li Q, Zhang Y, Xing Q, Han K, Song J, et al: Resveratrol exerts dosage-dependent effects on the self-renewal and neural differentiation of hUC-MSCs. Mol Cells 39: 418-425, 2016.

23. García-Zepeda SP, García-Villa E, Díaz-Chávez J, Hernández-Pando R and Gariglio P: Resveratrol induces cell death in cervical cancer cells through apoptosis and autophagy. Eur J Cancer Prev 22: 577-584, 2013.

24. Xiong W, Yin A, Mao X, Zhang W, Huang H and Zhang X Resveratrol suppresses human glioblastoma cell migration and invasion via activation of RhoA/ROCK signaling pathway. Oncol Lett 11: 484-490, 2016.

25. Kma L: Synergistic effect of resveratrol and radiotherapy in control of cancers. Asian Pac J Cancer Prev 14: 6197-6208, 2013.

26. Fang Y, DeMarco VG and Nicholl MB: Resveratrol enhances radiation sensitivity in prostate cancer by inhibiting cell proliferation and promoting cell senescence and apoptosis. Cancer Sci 103: 1090-1098, 2012

27. Liao HF, Kuo CD, Yang YC, Lin CP, Tai HC, Chen YY and Chen YJ: Resveratrol enhances radiosensitivity of human non-small cell lung cancer NCI-H838 cells accompanied by inhibition of nuclear factor-kappa B activation. J Radiat Res (Tokyo) 46: 387-393, 2005.

28. Lu KH, Chen YW, Tsai PH, Tsai ML, Lee YY, Chiang CY, Kao CL, Chiou SH, Ku HH, Lin CH, et al: Evaluation of radiotherapy effect in resveratrol-treated medulloblastoma cancer stem-like cells. Childs Nerv Syst 25: 543-550, 2009.

29. Chaussepied $M$ and Ginsberg D: Transcriptional regulation of AKT activation by E2F. Mol Cell 16: 831-837, 2004.

30. Korotayev K, Chaussepied $\mathrm{M}$ and Ginsberg D: ERK activation is regulated by E2F1 and is essential for E2F1-induced S phase entry. Cell Signal 20: 1221-1226, 2008. 